\title{
Transmesenteric hernia due to double-loop formation in the small intestine: a fatal case involving a toddler.
}

\section{AUTHOR(S):}

Kakimoto, Yu; Abiru, Hitoshi; Kotani, Hirokazu; Ozeki, Munetaka; Tsuruyama, Tatsuaki; Tamaki, Keiji

\section{CITATION:}

Kakimoto, Yu ... [et al]. Transmesenteric hernia due to double-loop formation in the small intestine: a fatal case involving a toddler.. Forensic science international 2012, 214(1-3): e39-e42

\section{ISSUE DATE:}

2012-01-10

URL:

http://hdl.handle.net/2433/152429

\section{RIGHT:}

(c) 2011 Elsevier Ireland Ltd.; この論文は出版社版でありません。引用の 際には出版社版をご確認ご利用ください。; This is not the published version. Please cite only the published version. 


\section{Case report}

Transmesenteric hernia due to double-loop formation in the small intestine: a fatal case involving a toddler

Yu Kakimoto, Hitoshi Abiru, Hirokazu Kotani, Munetaka Ozeki, Tatsuaki Tsuruyama, Keiji Tamaki

Department of Forensic Medicine and Molecular Pathology, Kyoto University,

Graduate School of Medicine, Yoshida-Konoe Sakyoku, Kyoto, 606-8501, Japan

Corresponding author. Tel.: +81 75753 4474; fax: +81757619591.

E-mail address: ktamaki@fp.med.kyoto-u.ac.jp (K. Tamaki) 


\section{Abstract}

We report a unique case of transmesenteric hernia resulting in death, which went undiagnosed during a recent hospital visit. The victim was a 2.5-year-old girl who-with the exception of chronic constipation—had no medical history. One night she complained of abdominal pains and was taken to a pediatric hospital where doctors performed an abdominal X-ray and echography. No significant findings suggesting bowel obstruction (e.g. air-fluid levels or dilation of the bowel) were obtained on examinations and bloody feces were not observed in this particular episode. As her abdominal pain gradually attenuated, the doctor allowed her to return home. A few hours later, she lost consciousness and expired despite resuscitation efforts attempted at an emergency hospital. A subsequent autopsy revealed that the small bowel had herniated through a defect in the mesentery resulting in two consecutive and inversely-forming loops, in which each loop protruded on either side of the mesentery. This rare morphological anatomy seems to have progressed in a two-step process. The girl's mild abdominal pain was likely induced by herniation and formation of the first intestinal loop, followed by severe shock occurring when the subsequent intestinal segment invaginated into the same defect forming the second loop on the opposite side of the mesentery. This case illustrates the difficulty of diagnosing transmesenteric hernia due to the presentation of unspecific symptoms; especially in infants and toddlers. Furthermore, this report demonstrates the value of a complete autopsy in cases of sudden and unexpected deaths involving children.

\section{Keywords}


transmesenteric hernia, strangulated hernia, intestinal obstruction, child, sudden death, forensic autopsy

\section{Introduction}

Internal hernia is defined as a protrusion of viscera through a mesenteric or peritoneal aperture within the abdominal and pelvic cavity. It is found in $0.2 \%$ to $0.9 \%$ of autopsy cases and $0.6 \%$ to $5.8 \%$ of small bowel obstruction cases [1,2]. The origins of these mesenteric and peritoneal defects are uncertain but most of the infant cases are regarded as congenital mechanisms. In contrast, adult cases occurring after gastrointestinal surgery—as represented by the Roux-en-Y procedure-are showing a growing trend in recent years [3,4].

Internal hernias are classified based on the location of the defect and are separated into six main groups: transmesenteric hernia, paraduodenal hernia, hernia through the foramen of Winslow, transomental hernia, pericecal hernia, and paravesical and pelvic hernia. Transmesenteric hernia is the most common type and is found in $60 \%$ to $83 \%$ of congenital internal hernias $[1,2,5]$.

Some internal hernias present with strangulation and these cases can be lethal if surgical intervention is not immediately undertaken. The mortality rate for internal hernias varies significantly from report to report, ranging from $2 \%$ to $30 \%$. Most of the cases ending in fatality involve the elderly who have underlying diseases: diabetes mellitus, chronic renal failure and gastrointestinal cancer with metastasis. The actual cause of death in these cases is post-operative sepsis $[1,2,6]$.

However, fatalities in children without severe complications (e.g. chromosomal aberrations and extremely low birth weight) have never before been reported $[7,8]$. In 
this report, we present a case of transmesenteric hernia resulting in the death of a toddler in the hopes that this rare emergent disease in children gains the attention it warrants.

\section{Case report}

\subsection{Clinical history}

A 2.5-year-old girl complained of abdominal pains one night after having dinner. As she often complained of constipation, her parents gave her an enema as usual. However, her pain persisted even after excreting soft feces, and so the parents took her to a local pediatric hospital. Upon arrival, she was apyretic and her vital signs were stable. Plain abdominal X-ray showed some ileac gas but no significant abnormalities indicating an ileus or perforation of the bowel. Echography was also performed but diagnostically sufficient images could not be obtained because the girl was uncooperative. Blood tests and computed tomography were not performed. While she stayed in the hospital, she sporadically excreted soft feces and occasionally vomited, however, bloody feces were not observed. After some time, her pain had attenuated and she stopped vomiting two hours after arriving at the hospital. The doctor suspected her condition as acute infectious gastroenteritis and allowed her to return home.

She continued to be in a tantrum at home and it took three hours to fall asleep. After approximately half-an-hour, she began vomiting again and this time lost consciousness. She was in cardiopulmonary arrest upon arrival at an emergency hospital, where she died despite two hours of resuscitation efforts.

The cause of the event remained unclear and she had no pertinent medical history or surgery before this episode. A forensic autopsy was performed 30 hours after death. 


\subsection{Autopsy findings}

The body showed normal development given the victim's age; her height was $96 \mathrm{~cm}$ (97 percentile) and weight was $13 \mathrm{~kg}$ (90 percentile of the average in Japan) [9]. There were no obvious superficial injuries on the surface of the body except for scars resulting from the medical treatments. The abdomen was soft and flat without distension.

Upon exploration, we identified a massive gangrenous segment of the small bowel in the peritoneal cavity (Fig.1a). The necrotic intestine had invaginated through an orifice of the mesentery near the ileocecum (Fig.1b). The ileum was strangulated and two consecutive and inversely forming loops were identified on opposite sides of the mesentery. The length of the oral loop was about $3 \mathrm{~cm}$ and the anal loop was $170 \mathrm{~cm}$. The diameter of the orifice was about $1.5 \mathrm{~cm}$ with smooth edges (Fig.1c). The total area of necrosis spanned from the ileum to the ileocecum (Fig.2) and filled with hemorrhagic fecal fluid. The ascending colon and the transverse colon contained hemorrhagic soft feces, while from the descending colon to the rectum, only yellow-brown soft feces were observed. No defects were identified in the intestinal ducts; however, about $300 \mathrm{ml}$ of bloody ascites was observed in the peritoneal cavity. In addition to this, $32 \mathrm{ml}$ and $10 \mathrm{ml}$ of bloody effusion were found in the right and left thoracic cavities, respectively. Malrotation of the bowel with midgut volvulus was not observed.

At histological examination, congestion was prominent in the herniated intestine and the submucosal layer swelled to 4 to 5 times the thickness of a normal intestine. The necrotic area was full of erythrocytes, and also occupied by a small number of inflammatory cells such as leukocytes or macrophages (Fig.3). Fibrin-layers outside the wall of the intestine upon the peritoneum were not formed. No other pathognomonic 
findings were observed in other viscera including the brain, heart, lungs, liver, or kidneys.

The cause of death was hypovolemic shock because she vomited frequently in the clinical history, and large amount of body fluid transferred to the peritoneal cavity and into the intestine. Although blood tests and bacterial culture were not performed, septic shock appeared less probable, considering her vital signs, the clear ascites and little inflammatory cells in other organs.

\section{Discussion}

Chronic constipation is one of the most common symptoms in childhood, but over $90 \%$ of the patients have no discernible organic cause [10]. The case presented in this manuscript is relatively misleading to a treating physician, considering the fact that abdominal pain is the most frequent complaint (66\%) in constipation [11]. As the mortality rate of gastrointestinal diseases in children is not as high as that of cardiovascular or respiratory disorders [12,13], abdominal pain without serious signs of shock is generally not given an adequate amount of consideration.

Some emergent cases involving gastrointestinal diseases include intussusception and gangrenous appendicitis; both of which can be lethal unless surgical intervention is promptly performed [14]. Transmesenteric hernias can also lead to life-threatening conditions, although not afforded the celebrity offered to the gastrointestinal diseases named above. Preoperative diagnosis of transmesenteric hernias is extremely challenging. In fact, most surviving cases were only diagnosed as a simple bowel obstruction prior to surgery [15-17]. Blood tests and ultrasonography are insufficient to evaluate organic pathology in pediatric abdominal disorders [18]. Some studies report 
the usefulness of CT scans to diagnose transmesenteric hernias and show the clustering of small bowel loops, although the mesenteric defect itself is not visualized [19-21]. Unfortunately, ordering CTs on all children who have ambiguous abdominal pain in busy clinical scenes is not a practical solution given the time-consuming nature of CT scans. Consequently it is often difficult to distinguish transmesenteric hernias from other benign illnesses in small children who show minimal symptoms and are not always cooperative with medical examinations.

In the present case, the ischemic intestine herniated through the mesenteric defect forming two distinctive loops on opposite sides of the mesentery. This morphology is consistent with the two-step clinical history the patient underwent (i.e. mild abdominal pain followed by sudden collapse). It is possible that circulation to the herniated area was narrowly maintained in the initial and gradual invagination of the intestine through the defect, which at first only caused slight abdominal pain. A few hours later, when the second, shorter internal segment herniated through the same defect-but on the opposite side-both segments of the intestine were completely mechanically strangulated by the small mesenteric defect, and this resulted in the immediate cut-off circulation to the herniated intestinal segments. In retrospect the initial hernia, which could most likely move in an out through the mesenteric defect with a certain degree of freedom, could have been the cause of the chronic constipation the victim suffered from throughout her lifetime. The final ischemic hernia occurred very acutely, as the hemorrhagic fluid did not move to the anal-side colon. Microscopic findings in the gangrened intestine also indicate the girl died before the inflammation expanded or fibrin precipitated on a systematic level. Together, these findings support the theory that the second, and critical, strangulation occurred when the second segment of the 
intestine herniated into the mesenteric defect after the toddler returned home.

This case demonstrates the difficulty of diagnosing transmesenteric hernias, as the condition involves considerable movement without the presence of a confining sac. Deterioration of the hernia may take various clinical courses depending on the size of the mesenteric defect and the morphology of the herniated intestine. In fact, this patient showed normal development for her age and never experienced a severe small bowel obstruction prior to this particular episode, regardless of the fact that the mesenteric defect probably existed from birth. It is important to note, however, that once the strangulated hernia is completely fixed, surgical treatment is the only viable treatment option.

In conclusion, open or laparoscopic explorations are the only way to establish a definitive anatomical diagnosis for transmesenteric hernias. Keeping in mind that this type of internal hernia may cause an acute bowel obstruction will aide physicians in deciding on a prompt surgical option of treatment without delay. As with any other potentially life threatening condition, bringing to light the precise cause of death via a thoroughly conducted postmortem examination enables us to learn from death so that this newly found knowledge may serve use in saving patients in the future.

\section{Acknowledgements}

We are very thankful to Dr. Richard H. Kaszynski for kindly providing professional editorial advice. 


\section{References}

[1] S. Ghiassi, S.Q. Nguyen, C.M. Divino, J.C. Byrn, A. Schlager: Internal hernias:clinical findings, management, and outcomes in 49 nonbariatric cases, $\mathrm{J}$ Gastrointest Surg 11 (2007) 291-295.

[2] H.P. Fan, A.D. Yang, Y.J. Chang, C.W. Juan, H.P. Wu: Clinical spectrum of internal hernia: a surgical emergency, Surg Today 38 (2008) 899-904.

[3] A. D'Haeninck, P. De Leyn, F. Swinnen: Internal herniation after Roux-en-Y gastric bypass: case reports and a review of the literature, Acta Chir Belg 109 (2009) 385-391.

[4] A. Rodriguez, M. Mosti, M. Sierra, R. Perez-Johnson, S. Flores, G. Dominguez, H. Sanchez, A. Zarco, K. Romay, M.F. Herrera: Small bowel obstruction after antecolic and antegastric laparoscopic Roux-en-Y gastric bypass: could the incidence be reduced?, Obes Surg 20 (2010) 1380-1384.

[5] A. Blachar, M.P. Federle: Internal hernia: an increasingly common cause of small bowel obstruction, Semin Ultrasound CT MR 23 (2002) 174-183.

[6] B.D. Newsom, J.S. Kukora: Congenital and acquired internal hernias: unusual causes of small bowel obstruction, Am J Surg 152 (1986) 279-285.

[7] D. Hirano: lleus due to transmesenteric hernia, Jpn J Pediatr Surg 39 (2007) $575-577$.

[8] R.W. Byard, R. Wick: Congenital mesenteric defects and unexpected death-a rare finding at autopsy, Pediatr Dev Pathol 11 (2008) 245-248.

[9] Ministry of Health, Labour and Welfare, Japan: Investigation of growth in childhood (2000). http://www.mhlw.go.jp/toukei/list/73-12.html

[10] B.R. Southwell, S.K. King, J.M. Hutson: Chronic constipation in children: organic 
disorders are a major cause, J Paediatr Child Health 41 (2005) 1-15.

[11] M.K. Miller, M.D. Dowd, M. Fraker: Emergency department management and short-term outcome of children with constipation, Pediatr Emerg Care 23 (2007) $1-4$

[12] R.W. Byard, A. Simpson: Sudden death and intussusception in infancy and childhood--autopsy considerations, Med Sci Law 41 (2001) 41-45.

[13] A.R. Tumer, L. Tumer, Y. Bilge: Sudden unexpected child deaths: forensic autopsy results in cases of sudden deaths during a 5-year period, J Trop Pediatr 51 (2005) 131-135.

[14] M. McCollough, G.Q. Sharieff: Abdominal surgical emergencies in infants and young children, Emerg Med Clin North Am 21 (2003) 909-935.

[15] C. Garignon, P. Paparel, R. Liloku, S. Lansiaux, T. Basset: Mesenteric hernia: a rare cause of intestinal obstruction in children, J Pediatr Surg 37 (2002) 1493-1494.

[16] Y.C. Ming, H.C. Chao, C.C. Luo: Congenital mesenteric hernia causing intestinal obstruction in children, Eur J Pediatr 166 (2007) 1045-1047.

[17] C.Y. Park, J.C. Kim, S.J. Choi, S.K. Kim: A transmesenteric hernia in a child: gangrene of a long segment of small bowel through a large mesenteric defect, Korean J Gastroenterol 53 (2009) 320-323.

[18] American Academy of Pediatrics Subcommittee on Chronic Abdominal Pain: Chronic abdominal pain in children, Pediatrics 115 (2005) 812-815.

[19] N. Takeyama, T. Gokan, Y. Ohgiya, S. Satoh, T. Hashizume, K. Hataya, H. Kushiro, M. Nakanishi, M. Kusano, H. Munechika: CT of internal hernias, Radiographics 25 (2005) 997-1015.

[20] L.C. Martin, E.M. Merkle, W.M. Thompson: Review of internal hernias: radiographic 
and clinical findings, AJR Am J Roentgenol 186 (2006) 703-717.

[21] G.A. Villalona, K.A. Diefenbach, R.J. Touloukian: Congenital and acquired mesocolic hernias presenting with small bowel obstruction in childhood and adolescence, J Pediatr Surg 45 (2010) 438-442. 
Legends for Figures

Fig.1

(a) Gangrenous small bowel.

(b) Transmesenteric hernia viewed after lifting the ileocecum. A $3 \mathrm{~cm}$ segment of the small bowel herniated in to the defect.

(c) Mesenteric defect $(1.5 \mathrm{~cm}$ diameter) with smooth edges.

\section{Fig.2}

Image of the entire intestinal tract and illustration showing the formation of intestinal loops. The short and long loops were approximately $3 \mathrm{~cm}$ and $170 \mathrm{~cm}$ in length, respectively. Arrows indicate the points where the intestine was strangulated by the mesenteric defect. 'O,' oral side; 'A,' anal side.

Fig.3 Microphotograph of the small bowel.

(a) Normal intestine. 4×, Azan stainig.

(b) Herniated intestine congested and swollen to 4-5 times normal thickness. 4x, Azan staining.

(c) Herniated intestine full of erythrocytes and virtually no inflammatory cells. 10x, Azan staining. 
Fig.1
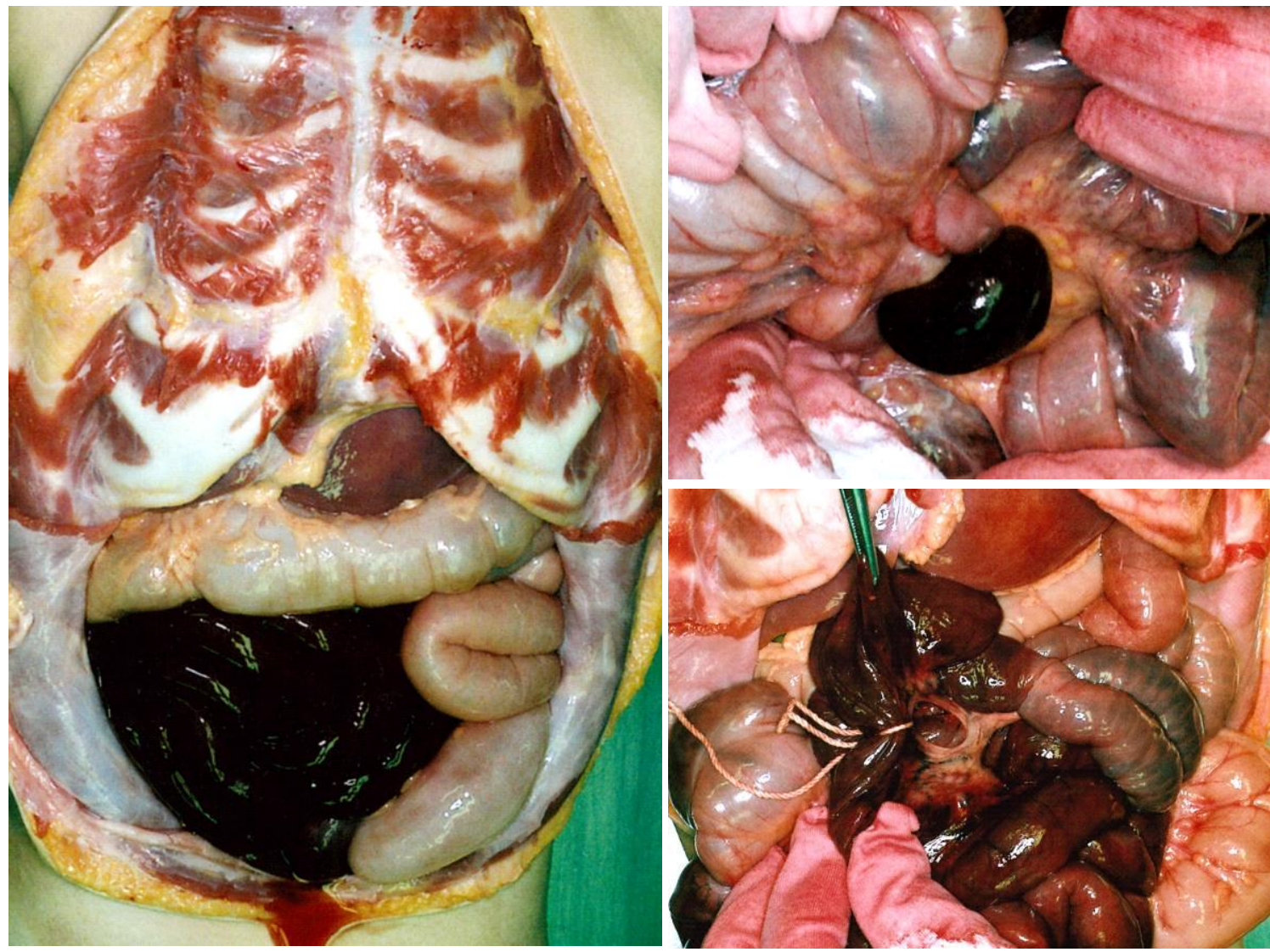
Fig.2

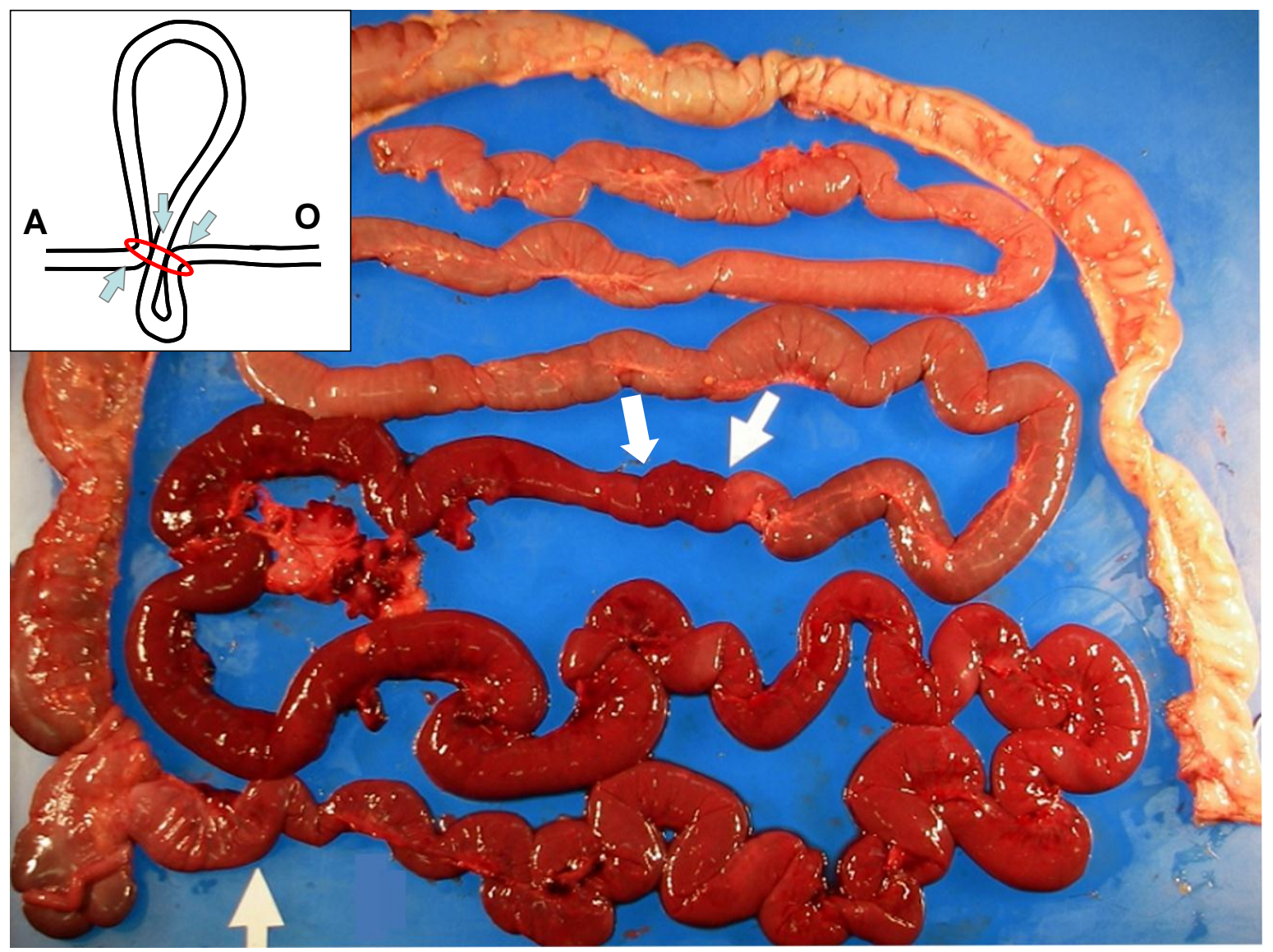


Fig.3

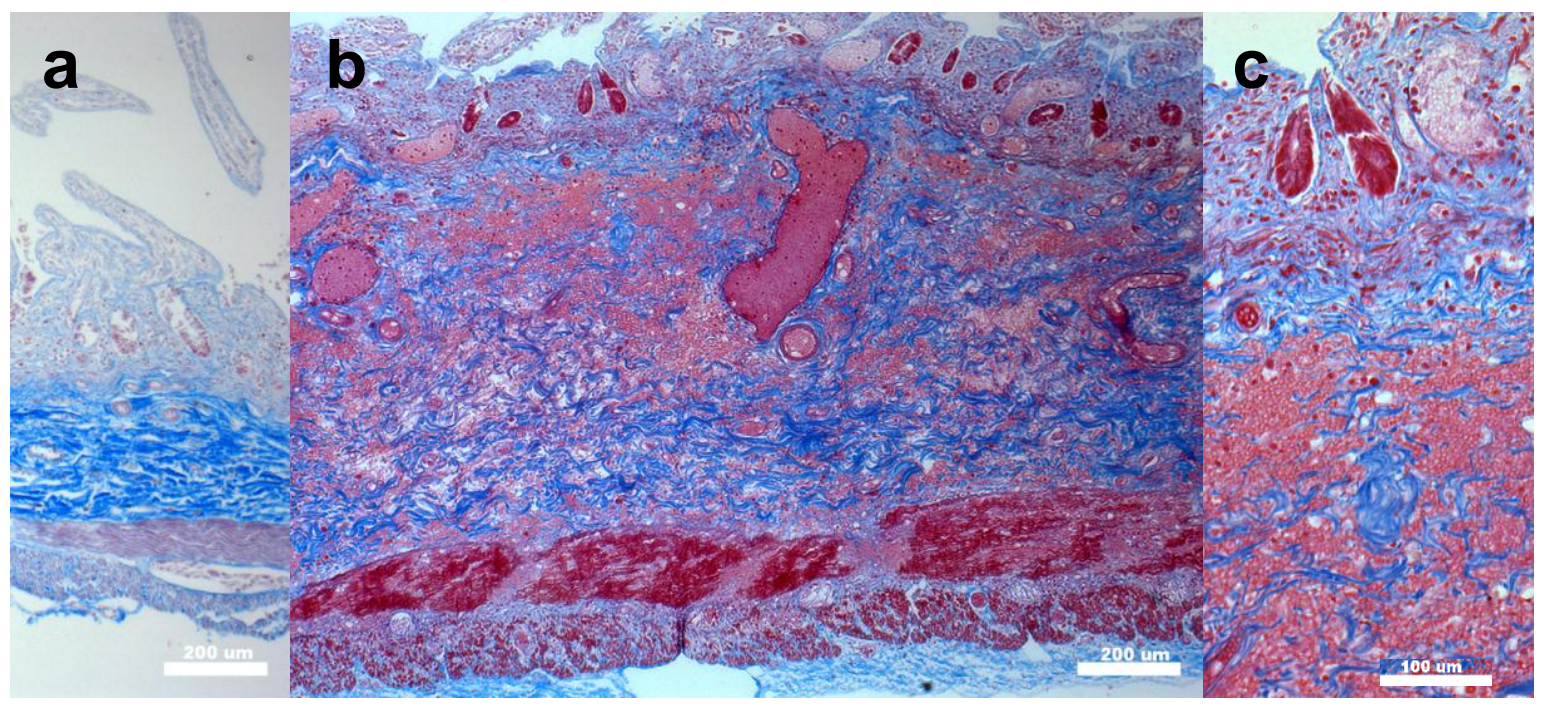

